\section{(2) OPEN ACCESS}

\title{
Effect of erenumab on functional outcomes in patients with episodic migraine in whom 2-4 preventives were not useful: results from the LIBERTY study
}

\author{
Michel Lanteri-Minet, ${ }^{1,2}$ Peter J Goadsby $10,{ }^{3}$ Uwe Reuter, ${ }^{4}$ Shihua Wen, ${ }^{5}$ \\ Peggy Hours-Zesiger, ${ }^{6}$ Michel D Ferrari, ${ }^{7}$ Jan Klatt (i) ${ }^{6}$
}

- Additional material is published online only. To view please visit the journal online (http://dx.doi.org/10.1136/ jnnp-2020-324396).

'Pain Department and FHU InovPain, CHU Nice - Côte Azur Université, Nice, France ${ }^{2}$ INSERM U1107 Migraine and Trigeminal Pain, Auvergne University, Clermont-Ferrand, France

${ }^{3}$ King's College London, NIHR/ Wellcome Trust King's CRF, London, UK

${ }^{4}$ Department of Neurology, Charite Universitatsmedizin Berlin, Berlin, Germany ${ }^{5}$ Novartis Pharmaceuticals Corp, East Hanover, New Jersey, USA ${ }^{6}$ Novartis Pharma AG, Basel, Switzerland ${ }^{7}$ Department of Neurology, Leiden University Medical Center, Leiden, the Netherlands

\section{Correspondence to} Dr Michel Lanteri-Minet, Pain Department and FHU InovPain, Centre Hospitalier Universitaire de Nice, Nice 06003, France; lanteri-minet.m@chu-nice.fr

Received 25 June 2020 Revised 10 November 2020 Accepted 8 December 2020 Published Online First 5 January 2021

Check for updates

(C) Author(s) (or their employer(s)) 2021. Re-use permitted under CC BY-NC. No commercial re-use. See rights and permissions. Published by BMJ.

To cite: Lanteri-Minet $\mathrm{M}$ Goadsby PJ, Reuter U, et al. J Neurol Neurosurg Psychiatry 2021:92:466-472.

\section{ABSTRACT}

Objective To evaluate the effect of erenumab on patient-reported, functional outcomes in patients with episodic migraine (EM) in whom 2-4 preventives were not useful from the Phase $3 \mathrm{~b}$ LIBERTY study.

Methods As previously reported, 246 patients with EM with 2-4 prior failed preventives were randomised 1:1 to subcutaneous erenumab $140 \mathrm{mg}$ or placebo every 4 weeks for 12 weeks. This analysis evaluated Migraine Physical Function Impact Diary (MPFID), Headache Impact Test (HIT-6) and Work Productivity and Activity Impairment (WPAI) scores at Week 12. P values were nominal without multiplicity adjustment.

Results Erenumab significantly improved MPFIDPhysical Impairment (PI) and Everyday Activities (EA) scores versus placebo (treatment difference (TD) $(95 \% \mathrm{CI})$ MPFID-PI: $-3.5(-5.7$ to -1.2$)(p=0.003)$; MPFID-EA: $-3.9(-6.1$ to -1.7$))(p<0.001)$ at 12 weeks. Patients on erenumab were more likely to have $a \geq 5$-point reduction in MPFID score (OR vs placebo $(95 \% \mathrm{CI})$ MPFID-EA: 2.1 (1.2 to 3.6); MPFID-PI: 2.5 (1.4 to 4.5)). A similar trend was observed for HIT-6 (TD: $-3.0 ; p<0.001)$; significantly higher proportions of patients on erenumab reported $a \geq 5$-point reduction (OR (95\% Cl): 2.4 (1.4 to 4.1)). In three out of four WPAI domains, erenumab showed improvement versus placebo.

Conclusion At 12 weeks, erenumab was efficacious on functional outcomes in patients with EM in whom 2-4 preventives were not useful.

Trial registration details ClinicalTrials.gov identifier: NCT03096834.

\section{INTRODUCTION}

Erenumab is a fully human monoclonal antibody that inhibits the canonical calcitonin gene-related peptide (CGRP) receptor. ${ }^{1}$ Clinical studies have demonstrated the efficacy and safety of erenumab in patients with episodic migraine $(\mathrm{EM})^{23}$ and chronic migraine $(\mathrm{CM})^{4}$ including in those with prior preventive migraine treatment failures. ${ }^{5} 6$ Results from the Phase $3 b$ LIBERTY study confirmed that erenumab is a potential treatment for the management of patients with EM in whom 2-4 preventives were not useful. ${ }^{7}$

An important component of migraine management is to evaluate headache-related functional impairment reported by patients and measured by patient-reported outcomes (PROs). ${ }^{8}$ The aim of this analysis was to evaluate the effect of erenumab versus placebo in patients in whom 2-4 preventives had not been useful from the Phase $3 b$ LIBERTY study on patient-reported, functional outcomes. These include outcomes assessing the impact of migraine on everyday activities and work productivity as well as those assessing, physical and functional impairment. An improvement in these areas indicates improved quality of life for patients.

\section{METHODS}

Standard protocol approvals, registrations and patient consents

The LIBERTY study is registered with ClinicalTrials.gov (NCT03096834). The final study protocol, informed consent form and accompanying materials provided to study patients were reviewed and approved by an independent ethics committee or relevant institutional review board at all participating sites. This study was conducted in accordance with International Council for Harmonisation Good Clinical Practice guidelines. All patients provided written informed consent. All centres complied with local regulations.

\section{Study design}

This analysis was based on data from the Phase 3b, 12-week, randomised, double-blind, placebocontrolled, multicentre, parallel group LIBERTY study conducted from 20 March 2017 until 27 October 2017, in 16 countries across Europe and Australia involving patients with EM in whom 2-4 preventives were not useful. The study design is reported elsewhere. ${ }^{7}$ Briefly, the study included a screening phase ( 0 to 2 weeks), baseline phase (4 weeks), double-blind treatment phase (12 weeks), an ongoing open-label treatment phase (156 weeks) and a safety follow-up phase (12 weeks).

Patients were randomised to receive placebo or erenumab $140 \mathrm{mg}$ subcutaneously in a $1: 1$ ratio, once every 4 weeks for 12 weeks. Patients who completed the 12-week double-blind treatment phase of the LIBERTY study were eligible to participate in an ongoing open-label treatment phase. The results of the extension phase will be reported separately. This article reports results from the 12 -week double-blind treatment phase.

Patients completed PRO questionnaires using an electronic diary (eDiary) platform. PRO questionnaires were administered to patients either at home 
or during visits to the clinic at baseline and during the doubleblind treatment phase.

\section{Inclusion criteria and previous preventive failures}

Patients aged 18 to 65 years, with EM (4 to 14 migraine days per month) ${ }^{9}$ were included in the LIBERTY study. ${ }^{7}$ Eligible patients had (1) failed 2-4 prior preventive treatments: propranolol/metoprolol, topiramate, flunarizine, valproate/divalproex, amitriptyline, venlafaxine, lisinopril, candesartan or other locally approved preventives (cinnarizine in the Czech Republic; indoramine in France; nadolol in Spain; oxetorone in France; and pizotifen in Austria, the Czech Republic, France, the Netherlands, Sweden and the UK); (2) failed or were not suitable for at least one of: propranolol/metoprolol, topiramate or flunarizine; and (3) failed or were not suitable for valproate/divalproex.

Efficacy failure was defined as no meaningful reduction in frequency of migraine attacks after administration of drugs for at least 2 to 3 months, ${ }^{10}$ at generally accepted therapeutic doses within the 5 years before screening. Tolerability failure was defined as documented discontinuation due to adverse events at any previous time. For the purposes of this study, being deemed unsuitable for treatment was defined as a patient being considered to be ineligible for a treatment for medical reasons such as contraindications or precautions included in local labels, national guidelines or other locally binding documents, or other medically relevant reasons as confirmed by the treating physician. Treatment failure and unsuitability were assessed on the basis of the patient's medical history and medical judgement. ${ }^{7}$

\section{Outcomes}

The primary and secondary efficacy endpoints of the LIBERTY study have been reported previously. ${ }^{7}$

\section{Migraine Physical Function Impact Diary}

Additional endpoints including change from baseline in scores on the everyday activities (EA) and physical impairment (PI) subdomains of the MigrainePhysical Function Impact Diary (MPFID), as well as the percentage of patients showing a $\geq 5$-point reduction in the MPFID-EA and MPFID-PI are evaluated in the current article. The MPFID is a self-administered, 13-item instrument measuring impact on EA (seven items) and PI (five items), with one stand-alone global question providing an assessment of overall impact on EA. ${ }^{11}$ Patients responded to items using a 5-point scale, with difficulty items ranging from 'without any difficulty' to 'unable to do' and frequency items ranging from 'none of the time' to 'all of the time'. These were assigned scores from 1 to 5 , with 5 representing the greatest burden. For each domain, the scores were calculated as the sum of the item responses and the sum was rescaled to a 0 to 100 scale, with higher scores representing higher burden. Patients with a reduction of 5 or more points in either of the two domains were considered to have shown a clinically meaningful reduction (improvement). ${ }^{12}$ Patients completed the MPFID every day using the eDiary, with a recall period of the past 24 hours.

\section{Headache Impact Test}

HeadacheImpact Test (HIT-6) is a short-form self-administered questionnaire based on the Internet-HIT question pool that evaluates how often headaches affect activities or cause distress, using the functionally relevant domains. ${ }^{13}$ Six domains assess the frequency of pain severity, headaches limiting daily activity (household, work, school and social), wanting to lie down when headache is experienced, feeling too tired to work or do daily activities because of headache, feeling 'fed up' or irritated because of headache and headaches limiting ability to concentrate or work on daily activities. No recall period is specified for the first three items, while the past 4 weeks is used for the last three items. HIT-6 scores are categorised into four grades, representing little or no impact (49 or less), some impact (50 to 55), substantial impact (56 to 59) and severe impact (60 to 78 ) due to headache; higher scores suggest negative impact. The thresholds for minimally important intergroup differences are well established; -1.5 for $\mathrm{EM}^{14}$ and -2.3 for $\mathrm{CM}^{15}$ In an individual patient, a reduction of 5 points is usually considered clinically meaningful with negative change indicating improvement. ${ }^{16}$

Functional impairment in patients with migraine was measured using change in HIT-6 score from baseline to Week 12, and percentage of patients with $\mathrm{a} \geq 5$-point reduction in total HIT- 6 score between baseline and Week 12 .

\section{Work Productivity and Activity Index}

The Work Productivity and Activity Index (WPAI) (change from baseline at Week 12), a commonly used scale that assesses work productivity and economic consequences of various disease states, was also evaluated. Although a headache-specific version exists, few trials have used the WPAI to assess the effects of preventive treatment. ${ }^{17}$

In the WPAI-Headache tool, patients are asked six questions about work and activity impairment due to headache, including hours worked and hours missed in the last 7 days. WPAI scores are based on 1-item (presenteeism, activity impairment), 2-items (absenteeism) and multiple items (overall work productivity); a score cannot be calculated if there is a missing response to the corresponding item. The questionnaire was collected weekly and took approximately $10 \mathrm{~min}$ to complete at home. Due to the design of the questionnaire, patients who were not employed or who were employed but with extensive 'no-work status' were not included in the analysis. Although, clinically meaningful change with respect to the WPAI scores particularly for migraine have not been determined, higher WPAI scores indicate greater impairment and negative change in the scores indicate an improvement in a particular work productivity domain.

\section{Statistical analysis}

In the LIBERTY study, the randomised analysis set included all randomised patients and was used to summarise patient disposition, demographics and baseline disease characteristics. The full analysis set included all randomised patients who initiated the study medication and completed at least one post-baseline monthly migraine days (MMD) measurement in the double-blind treatment phase. Data from the full analysis set was analysed based on preplanned randomised treatment. The full analysis set was used to summarise efficacy endpoints. Main analyses were done over Weeks 9 to 12 .

Demographic variables and other baseline characteristics were summarised using descriptive statistics by randomised treatment group and overall study population. All continuous endpoints (ie, change from baseline) were analysed using a linear longitudinal mixed effects model including treatment group, baseline value, stratification factor, scheduled visit and the interaction of treatment group with scheduled visit, without any imputation for missing data. Unstructured covariance matrix structure was assumed. The dichotomous endpoints derived from corresponding continuous endpoints were analysed using the stratified Cochran-Mantel-Haenszel test after imputing missing data as non-response (as for the primary endpoint). Estimates 
Migraine

Table 1 Key baseline and demographic characteristics of the enrolled patients in the LIBERTY study (randomised set)

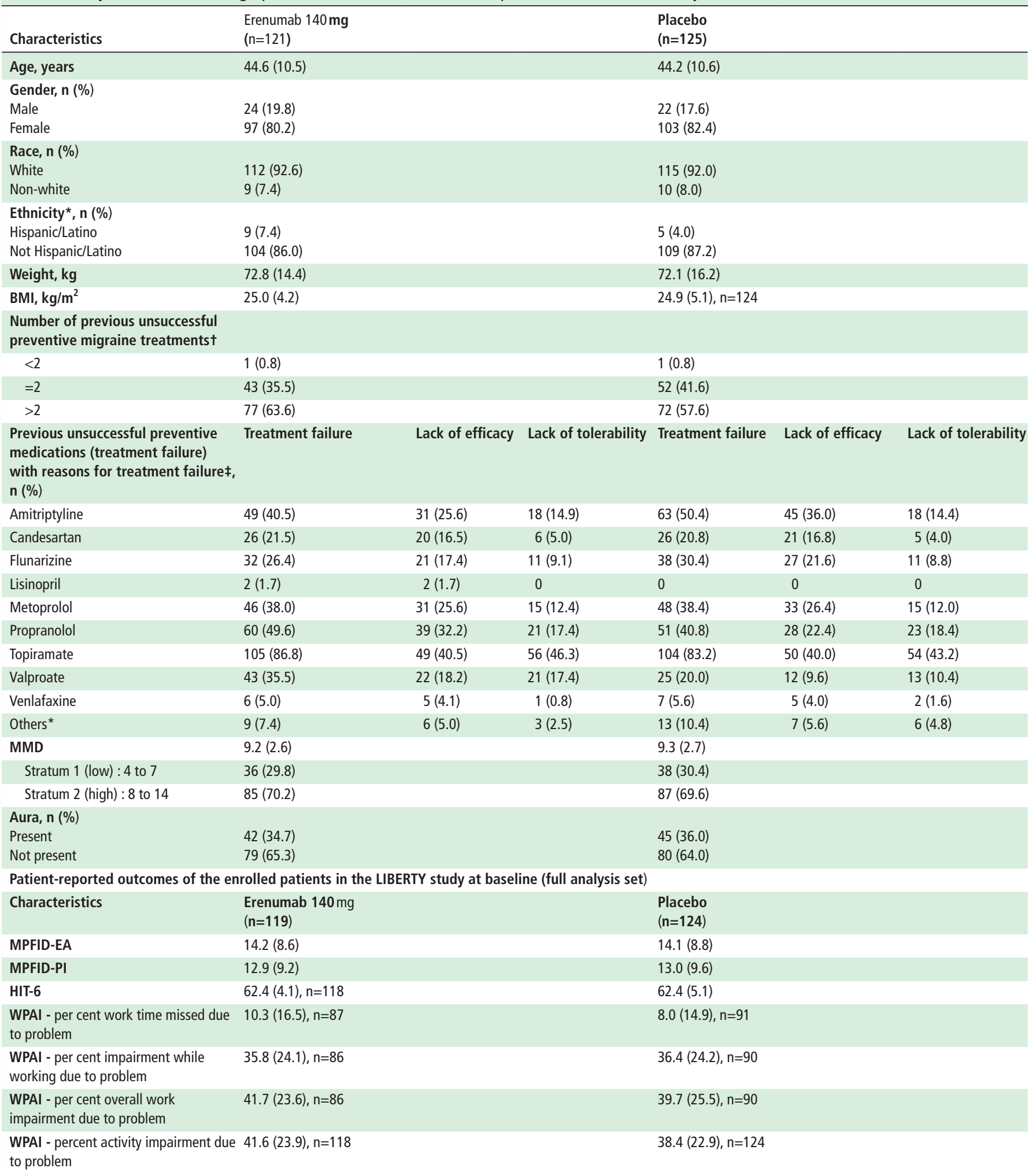

\section{Data are mean (SD) or $\mathrm{n}(\%)$.}

${ }^{*}$ Ethnicity data were missing for 8 patients in the erenumab group and 11 in the placebo group.

tOne patient in each group had unsuccessfully used fewer than two preventive treatments.

$\ddagger$ Does not include patients considered unsuitable for treatment.

§Includes cinnarizine, indoramin, nadolol, oxetorone and pizotifen.

BMI, body mass index; HIT-6, Headache Impact Test; MPFID-EA, Migraine Physical Function Impact Diary-Everyday Activities; MPFID-PI, Migraine Physical Function Impact DiaryPhysical Impairment; $\mathrm{N}$, number of patients included in the analysis set; $\mathrm{n}$, number of patients for which data was available at baseline; WPAl, Work Productivity and Activity Impairment. 
Table 2 Proportion of patients with a $\geq 5$-point reduction from baseline in MPFID-PI and MPFID-EA score (full analysis set)

\begin{tabular}{|c|c|c|c|c|c|c|c|c|}
\hline Week 4 & & & Week 8 & & & Week 12 & & \\
\hline $\begin{array}{l}\text { Erenumab } \\
140 \mathrm{mg} \\
(\mathrm{n}=119)\end{array}$ & $\begin{array}{l}\text { Placebo } \\
(n=124)\end{array}$ & \multirow{2}{*}{$\begin{array}{l}\text { OR }(95 \% \mathrm{Cl}) \text {, } \\
\text { P value }\end{array}$} & $\begin{array}{l}\text { Erenumab } \\
140 \mathrm{mg} \\
(\mathrm{n}=119)\end{array}$ & $\begin{array}{l}\text { Placebo } \\
(n=124)\end{array}$ & \multirow{2}{*}{$\begin{array}{l}\text { OR }(95 \% \mathrm{Cl}) \text {, } \\
\text { P value }\end{array}$} & $\begin{array}{l}\text { Erenumab } \\
140 \mathrm{mg} \\
(\mathrm{n}=119)\end{array}$ & $\begin{array}{l}\text { Placebo } \\
(n=124)\end{array}$ & \multirow{2}{*}{$\begin{array}{l}\text { OR }(95 \% \mathrm{Cl}) \text {, } \\
\text { P value }\end{array}$} \\
\hline $\mathrm{n} / \mathrm{N}(\%)$ & & & $\mathrm{n} / \mathrm{N}(\%)$ & & & $\mathrm{n} / \mathrm{N}(\%)$ & & \\
\hline \multicolumn{9}{|l|}{ MPFID-PI } \\
\hline $\begin{array}{l}36 / 119 \\
(30.3)\end{array}$ & 15/124 (12.1) & $3.3(1.6$ to 6.4$),<0.001^{*}$ & $39 / 119(32.8)$ & $14 / 124(11.3)$ & $\begin{array}{l}3.8 \text { (2.0 to } \\
7.6),<0.001 \text { * }\end{array}$ & $45 / 119(37.8)$ & $24 / 123(19.5)$ & 2.5 (1.4 to 4.5$), 0.002^{*}$ \\
\hline \multicolumn{9}{|l|}{ MPFID-EA } \\
\hline $\begin{array}{l}42 / 119 \\
(35.3)\end{array}$ & 21/124 (16.9) & $2.7(1.5$ to 5.0$), 0.001^{*}$ & $51 / 119(42.9)$ & $26 / 124(21.0)$ & $\begin{array}{l}2.8(1.6 \text { to } \\
5.0),<0.001 *\end{array}$ & $49 / 119(41.2)$ & $31 / 123(25.2)$ & 2.1 (1.2 to 3.6$), 0.010^{*}$ \\
\hline
\end{tabular}

Statistical analysis used a Cochran-Mantel-Haenszel test adjusted for stratification factor (4 to 7 vs 8 to 14 migraine days at baseline) after missing data were imputed as nonresponse. *Indicates statistical significance (two-sided) at a 0.05 alpha level.

MPFID-EA, Migraine Physical Function Impact Diary-Everyday Activities; MPFID-PI, Migraine Physical Function Impact Diary-Physical Impairment; N, number of patients included in the analysis set; ;n, number of patients who responded.

(treatment difference or OR) of erenumab compared with placebo, with associated $95 \%$ CIs and $\mathrm{p}$ values were provided.

No multiplicity adjustment was performed in this study. In addition, given that the subgroup analysis and the analyses of the PROs discussed in this manuscript were run as exploratory analysis in the study, all statistical significances claimed in this manuscript where $p$ values are $<0.05$ were considered nominal without further specification. Statistical analysis of all data was performed using SAS statistical software V.9 (SAS Institute, Cary, North Carolina, USA).

\section{Classification of evidence}

The study provides Class I evidence that for patients with EM who have failed 2-4 prior preventive treatments, erenumab showed favourable treatment effects on PRO scales and was more efficacious than placebo irrespective of age, gender, MMD and number of prior preventive treatment failures.
Data availability

The data for the analyses described in this report are available by request from the author investigators or Novartis and/or Amgen, the companies sponsoring the clinical development of erenumab for the treatment of migraine.

\section{RESULTS}

Patient disposition, baseline characteristics and primary results

In the LIBERTY study, a total of 246 patients were randomised to receive placebo $(n=125)$ or erenumab $140 \mathrm{mg}(n=121)$. Of these, $240(97.6 \%)$ completed the double-blind treatment phase. The treatment groups were generally well balanced in terms of baseline demographic and disease characteristics (table 1).

Table 1 also details the PROs of patients at baseline. At Week $12,36(30.0 \%)$ patients in the erenumab group had $a \geq 50 \%$

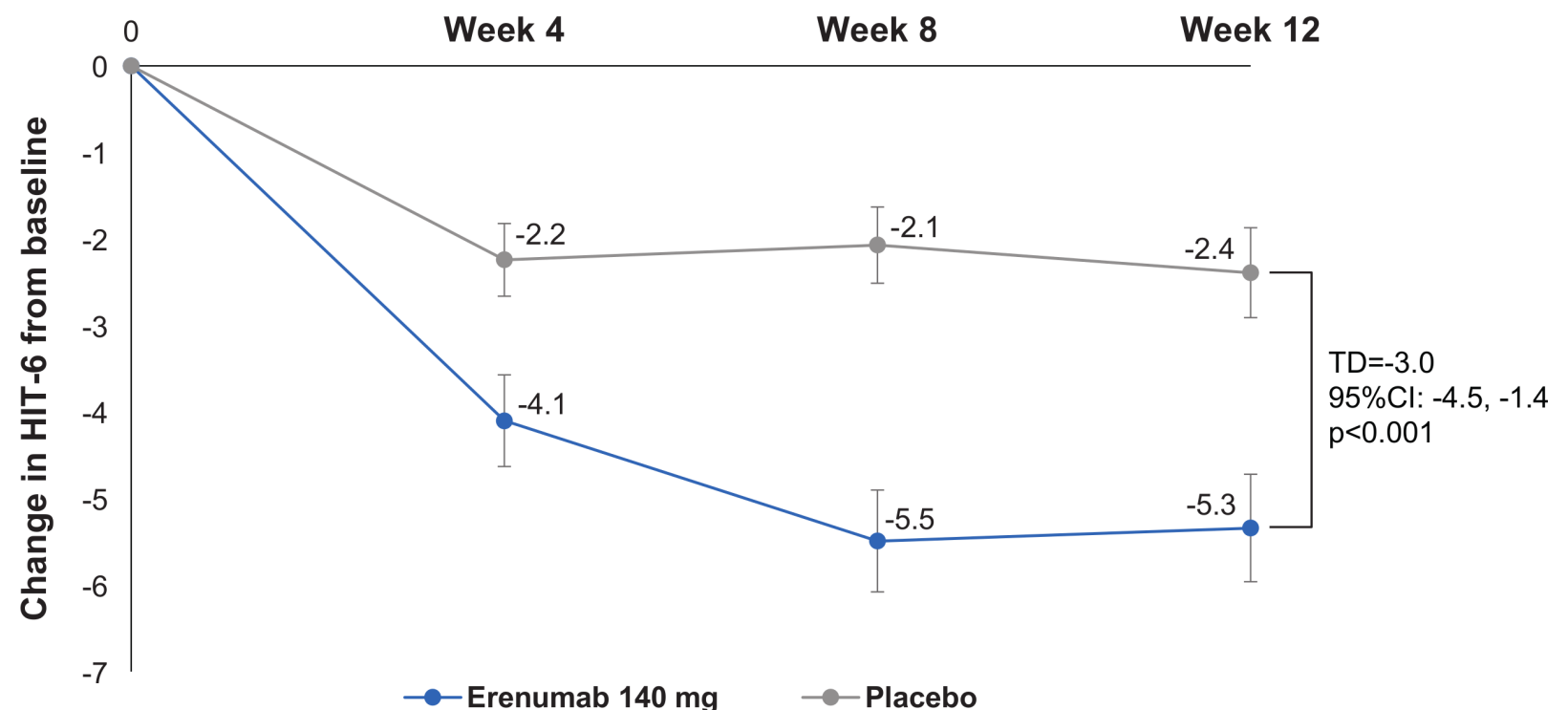

\begin{tabular}{lccc} 
& & & \\
& Week 4 & Erenumab 140 mg & - Placebo \\
& $-1.9(-3.1,-0.6)$ & $-3.4(-4.8,-2.0)$ & Week 12 \\
\hline TD (95\% CI) & 0.003 & $<0.001$ & $-3.0(-4.5,-1.4)$ \\
\hline p value & & & $<0.001$
\end{tabular}

Figure 1 Mean change from baseline in HIT-6. Adjusted mean change (SE) reported. HIT-6, Headache Impact Test; TD, treatment difference. 
Table 3 Proportion of patients with $\mathrm{a} \geq 5$-point reduction from baseline in HIT-6 total score (full analysis set)

\begin{tabular}{|c|c|c|c|c|c|c|c|c|}
\hline Week 4 & & & Week 8 & & & Week 12 & & \\
\hline Erenumab & & \multirow{3}{*}{$\begin{array}{l}\text { OR }(95 \% \mathrm{Cl}) \text {, } \\
\text { P value }\end{array}$} & Erenumab & & \multirow{3}{*}{$\begin{array}{l}\text { OR }(95 \% \mathrm{Cl}) \text {, } \\
\mathrm{P} \text { value }\end{array}$} & Erenumab & & \multirow{3}{*}{$\begin{array}{l}\text { OR }(95 \% \mathrm{Cl}), \\
\mathrm{P} \text { value }\end{array}$} \\
\hline $\begin{array}{l}140 \mathrm{mg} \\
(\mathrm{n}=119)\end{array}$ & $\begin{array}{l}\text { Placebo } \\
(n=124)\end{array}$ & & $\begin{array}{l}140 \mathrm{mg} \\
(\mathrm{n}=119)\end{array}$ & $\begin{array}{l}\text { Placebo } \\
(n=124)\end{array}$ & & $\begin{array}{l}140 \mathrm{mg} \\
(\mathrm{n}=119)\end{array}$ & $\begin{array}{l}\text { Placebo } \\
(n=124)\end{array}$ & \\
\hline $\mathrm{n} / \mathrm{N}(\%)$ & & & $\mathrm{n} / \mathrm{N}(\%)$ & & & $\mathrm{n} / \mathrm{N}(\%)$ & & \\
\hline $45 / 119(37.8)$ & $31 / 124(25.0)$ & 1.8 (1.1 to 3.1 ) $0.032^{*}$ & $56 / 119(47.1)$ & $30 / 124(24.2)$ & $\begin{array}{l}2.8 \text { (1.6 to } \\
4.8)<0.001^{*}\end{array}$ & $55 / 119(46.2)$ & $33 / 124(26.6)$ & 2.4 (1.4 to 4.1$) 0.002^{*}$ \\
\hline
\end{tabular}

Statistical analysis used a Cochran-Mantel-Haenszel test adjusted for stratification factor (4 to 7 vs 8 to 14 migraine days at baseline) after missing data were imputed as nonresponse. *Indicates statistical significance (two-sided) at a 0.05 alpha level.

HIT-6, Headache Impact Test; N, number of patients included in the analysis set; ;n, number of patients who responded.

reduction from baseline in mean MMD, compared with 17 $(14.0 \%)$ in the placebo group (OR (95\% CI): 2.7 (1.4 to 5.2); $\mathrm{p}=0.002)$. All secondary endpoints were met. The tolerability and safety profiles of erenumab and placebo were similar. ${ }^{7}$

\section{PATIENT-REPORTED OUTCOMES}

\section{Migraine Physical Function Impact Diary}

Patients treated with erenumab showed significantly better MPFID-PI and -EA scores compared with those treated with placebo. The mean change (SD) from baseline at Week 12 in MPFID-PI was $-1.9(0.8)$ for the erenumab group and +1.6 $(0.8)$ for the placebo group, with a difference between treatments of $-3.5(-5.7$ to $-1.2 ; \mathrm{p}=0.003)$. The corresponding values for the MPFID-EA domain were -3.4 (0.8) for the erenumab group and $+0.6(0.8)$ for the placebo group, with a difference between treatments of $-3.9(-6.1$ to $-1.7 ; \mathrm{p}<0.001)$.

The change from baseline in PI and the impact of erenumab on EA as measured by the MPFID were both significant at all time points (ie, at Weeks 4, 8 and 12) with a mean reduction in both scores in the erenumab $140 \mathrm{mg}$ treatment group compared with a mean increase in both scores in the placebo group at Week $12^{7}$

At Week 12, the proportion of patients with a $\geq 5$-point reduction from baseline in MPFID-EA (OR (95\% CI): 2.1 (1.2 to 3.6)) and MPFID-PI score (OR (95\% CI): 2.5 (1.4 to 4.5)) were higher with erenumab compared with placebo (table 2 ).

\section{Headache Impact Test}

There was a significantly greater mean reduction in the change in the daily impact of headache as measured by the HIT-6, from baseline to Week 12 and at each of the earlier visits (Weeks 4 and 8) evident from Week 4 (first time-point of assessment) for erenumab $140 \mathrm{mg}$ compared with placebo (figure 1).

At Week 12, 55 of $119(46.2 \%)$ patients in the erenumab group had $a \geq 5$ point reduction in HIT-6 total score from baseline which was nearly twofold higher compared with 33 of 124 $(26.6 \%)$ patients in the placebo group (OR $(95 \% \mathrm{CI}): 2.4$ (1.4 to 4.1); $\mathrm{p}=0.002$; table 3).

A significantly greater proportion of patients in the erenumab group than in the placebo group also had a $\geq 5$-point reduction in HIT-6 total score from baseline at Weeks 0 to 4 and Weeks 5 to 8 (table 3 ).

\section{Work Productivity and Activity Impairment}

Patients who were not working were excluded from the analysis of WPAI. There were favourable trends in patients treated with erenumab $(n=119)$ versus placebo $(n=124)$ among all parameters measured by the WPAI at each time point of assessment.

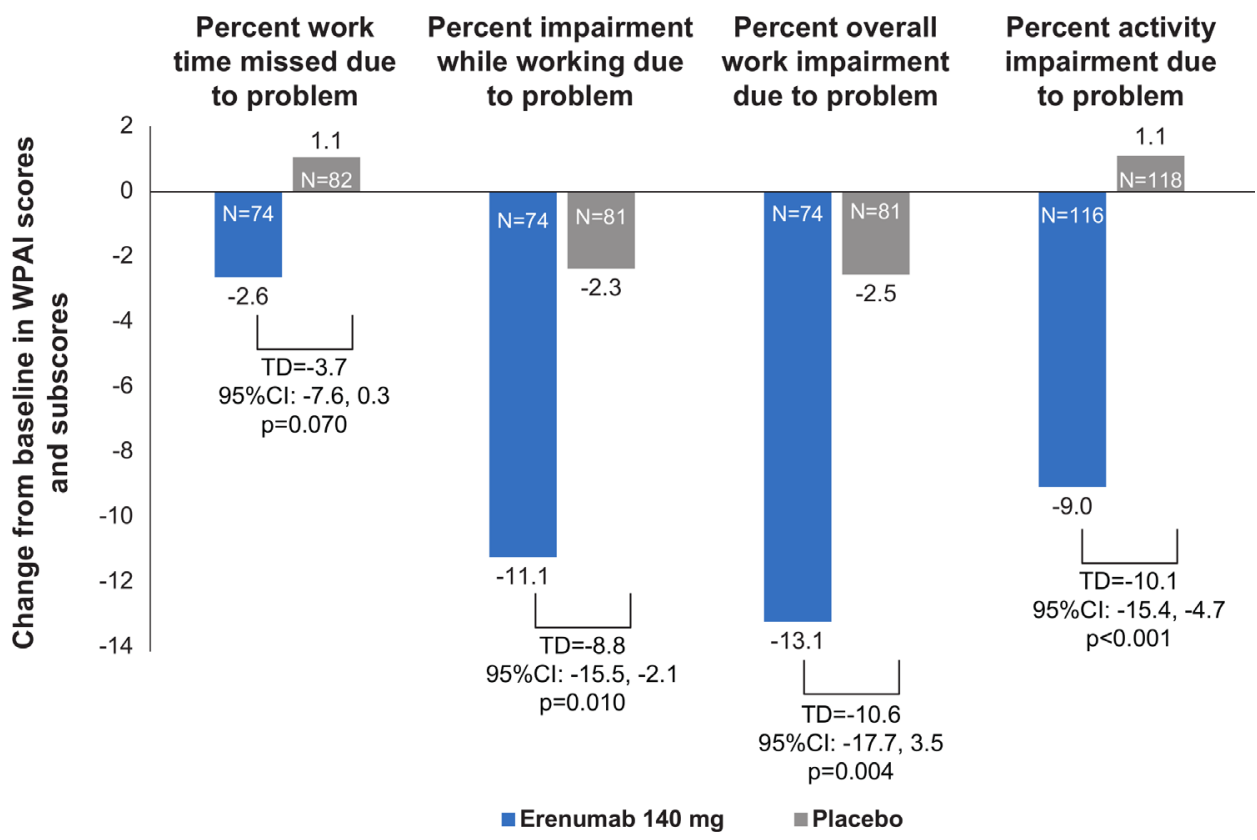

Figure 2 Change from baseline in WPAl scores and subscores at Week 12. Adjusted mean reported. A negative change in WPAI score indicates an improvement in the work productivity domain. TD, treatment difference; WPAl, Work Productivity and Activity Impairment. 
There were significant improvements in three out of four parameters for patients on erenumab at Week 12, compared with placebo $(p<0.05)$. Improvements were seen in the per cent impairment while working due to problem, per cent overall work impairment due to problem and per cent activity impairment due to a problem (figure 2).

\section{DISCUSSION}

The phase $3 \mathrm{~b}$ LIBERTY study assessed the efficacy and safety of a CGRP-directed therapy in a population with a high unmet need, in whom 2-4 preventives had not been useful. ${ }^{7}$ The PRO data reported here examined the short-term effects of erenumab on functioning and work productivity in patients with EM who failed prior preventive treatments. Significant differences in change from baseline in HIT-6 and MPFID-EA and MPFID-PI scores, favoured erenumab-treated patients versus placebo. Improvement was observed in patients treated with erenumab versus placebo in a majority of the parameters measured by the WPAI at each assessment time point (Weeks 4, 8 and 12). These findings confirm that the efficacy observed in traditional parameters such as MMD, migraine-specific medication treatment days and response rates translate into functional improvement. Of note, this improvement was observed in different domains and aspects, such as overall impact (HIT-6), PI and EA (MPFID) and work-related disability (WPAI). All of these instruments assess different and complementary aspects that are highly relevant for patients and provide complementary information in the evaluation of erenumab as a migraine preventive treatment.

The societal burden of migraine is increasing, especially with increasing indirect costs relating to reduced work productivity and absenteeism. ${ }^{18}$ Impaired functioning and disability among patients with migraine is high, indicating a high unmet medical need for agents that achieve treatment goals and at the same time improve quality of life. ${ }^{19}$ With an ongoing research on newer preventive migraine treatments, it is extremely important to achieve treatment goals with a constant urge to improve patient functioning. Post hoc analysis on data from a Phase $2 \mathrm{~b}$ study that evaluated subcutaneous galcanezumab $120 \mathrm{mg}$ every 28 days versus placebo was associated with significant patientreported improvements in functioning as measured by MigraineSpecific Quality of Life Questionnaire. ${ }^{20}$ However, the HIT-6 scores were not associated with significant functional improvement following 12 weeks of galcanezumab treatment. Another global, 6-month, Phase 3 study evaluated subcutaneous galcanezumab $120 \mathrm{mg}$, galcanezumab $240 \mathrm{mg}$ and placebo. ${ }^{21}$ Treatment with galcanezumab was associated with reduced functional impairment as measured in the Migraine-Specific Quality of Life Questionnaire Role Function-Restrictive, improvement in patients' global impression of severity of their disease assessed by Patients Global Impression of Severity rating and improvement in the total Migraine Disability Assessment scores compared with placebo. ${ }^{21}$ However, as patient inclusion and exclusion criteria differed between the different studies, the outcomes cannot be compared directly. A recent review article studied calcitonin gene related peptide monoclonal antibodies (CGRP$\mathrm{mABs}$ ) efficacy on improving the quality of life and decreasing disability and impact of migraine measured using patient-related outcomes, on patients who participated in clinical trials with erenumab, fremanezumab, galcanezumab and eptinezumab. ${ }^{22}$ Evidence suggested that CGRP-mABs had an impact on the disability, workplace productivity and quality of life, all of which will translate into changes in the real world of migraine patients who are treated with CGRP-mABs. ${ }^{22}$ The results of our study are in line with the observations of CGRP-mABs.

\section{Study limitations}

The study was limited to patients with EM and therefore, the results should not be generalised to include those with $\mathrm{CM}$, even though the number of MMD are within the range for the $\mathrm{CM}$ definition. The short duration of the study limits the correlation of long-term treatment with erenumab with patient functioning, though an ongoing, 3-year open-label extension will provide additional information. For the analysis of WPAI, the sample size for this endpoint was lower due to some patients not being in work (and therefore being excluded from the WPAI analysis). This low sample size could be a possible reason for a statistically significant difference between erenumab and placebo not being observed for all WPAI subscores.

\section{CONCLUSION}

In these analyses of the Phase 3b LIBERTY study, patients receiving erenumab $140 \mathrm{mg}$ showed significantly higher levels of improvement of migraine-related functional and physical impairment, improved work productivity, improvement in everyday activities and patients' lives, compared with those taking placebo. These findings, obtained via the use of PROsthe MPFID-EA and PI, HIT-6, and WPAI questionnaires-corroborate the efficacy observed with erenumab in LIBERTY on traditional measures of treatment efficacy (such as number of migraine days).

Efficacy on PROs appeared to be of rapid onset, with differences versus placebo observed at all time points of assessment. These benefits were sustained throughout the 12 -week doubleblind treatment phase.

The findings of these analyses add to previous erenumab efficacy and safety data obtained from the LIBERTY study. They show that erenumab exerts a positive effect on patient functioning and work productivity, as assessed by PRO measures among patients with EM in whom 2-4 preventives had not been useful.

Correction notice This article has been corrected since it first published. The provenance and peer review statement has been included.

Acknowledgements The authors thank the LIBERTY study participants and investigators for their commitment to this study. The authors also thank Aditi Kataria of Novartis Healthcare Pvt Ltd for providing medical writing support/editorial support, which was funded by Novartis Pharma AG, Basel, Switzerland, in accordance with Good Publication Practice (GPP3) guidelines (http://www.ismpp.org/gpp3).

Contributors UR, PJG and JK participated in the design of the study. The chief investigators were UR, PJG, ML-M and MDF. PH-Z participated in patient data collection. SW was the study biostatistician responsible for the statistical analyses. All authors were involved in interpretation of the data. All authors agreed on the content of the manuscript, reviewed drafts and approved the final version.

Funding The complete study was supported by Novartis Pharma AG, Basel, Switzerland.

Competing interests ML-M received grants and honoraria for advisory boards, speaker panels or investigation studies from Allergan, Amgen, Astellas, ATI, BMS, Boehringer, Boston Scientific, CoLucid, Convergence, GlaxoSmithKline, Grunenthal, Eli Lilly, Medtronic, Menarini, MSD, Novartis, Pfizer, Reckitt Benckiser, Saint-Jude, Sanofi-Aventis, Teva Pharmaceuticals, UCB and Zambon; PJG, received grants and personal fees from Amgen and Eli Lilly; personal fees from Alder Biopharmaceuticals, Allergan, Autonomic Technologies Inc, Dr Reddy's Laboratories, Electrocore, eNeura, MundiPharma, Novartis, Teva Pharmaceuticals, Trigemina Inc, WL Gore, MedicoLegal work, Massachusetts Medical Society, Up-to-Date, Oxford University Press and Wolters Kluwer; and a patent for magnetic stimulation for headache assigned to eNeura without fee; UR received consulting fees, speaking/teaching fees and/ or research grants from Allergan, Amgen, Autonomic Technologies, CoLucid, ElectroCore, Eli Lilly, Medscape, Novartis, StreamMedUp and Teva Pharmaceuticals; SW, PH-Z and JK are employees of, and hold stocks in, Novartis; MDF received 
grants, consultancy or trial support from Medtronic, ElectroCore, Amgen, Eli Lilly, Teva Pharmaceuticals and Novartis, and independent support from the European Community, NWO, NIH and the Dutch Heart Foundation.

Patient consent for publication Not required.

Provenance and peer review Not commissioned; externally peer reviewed.

Data availability statement Data are available upon reasonable request. All data relevant to the study are included in the article or uploaded as supplementary information. The data for the analyses described in this report are available by request from the author investigators or Novartis and/or Amgen, the companies sponsoring the clinical development of erenumab for the treatment of migraine.

Open access This is an open access article distributed in accordance with the Creative Commons Attribution Non Commercial (CC BY-NC 4.0) license, which permits others to distribute, remix, adapt, build upon this work non-commercially, and license their derivative works on different terms, provided the original work is properly cited, appropriate credit is given, any changes made indicated, and the use is non-commercial. See: http://creativecommons.org/licenses/by-nc/4.0/.

\section{ORCID iDs}

Peter I Goadsby http://orcid.org/0000-0003-3260-5904

Jan Klatt http://orcid.org/0000-0002-2276-4701

\section{REFERENCES}

1 Shi L, Lehto SG, Zhu DXD, et al. Pharmacologic characterization of AMG 334, a potent and selective human monoclonal antibody against the calcitonin gene-related peptide receptor. J Pharmacol Exp Ther 2016;356:223-31.

2 Dodick DW, Ashina M, Brandes JL, et al. Arise: a phase 3 randomized trial of erenumab for episodic migraine. Cephalalgia 2018:38:1026-37.

3 Goadsby PJ, Reuter U, Hallström Y, et al. A controlled trial of erenumab for episodic migraine. N Engl J Med 2017;377:2123-32.

4 Tepper S, Ashina M, Reuter U, et al. Safety and efficacy of erenumab for preventive treatment of chronic migraine: a randomised, double-blind, placebo-controlled phase 2 trial. Lancet Neurol 2017;16:425-34.

5 Ashina M, Tepper S, Brandes JL, et al. Efficacy and safety of erenumab (AMG334) in chronic migraine patients with prior preventive treatment failure: a subgroup analysis of a randomized, double-blind, placebo-controlled study. Cephalalgia 2018:38:1611-21.

6 Goadsby PJ, Paemeleire K, Broessner G, et al. Efficacy and safety of erenumab (AMG334) in episodic migraine patients with prior preventive treatment failure: a subgroup analysis of a randomized, double-blind, placebo-controlled study. Cephalalgia 2019:39:817-26.
7 Reuter U, Goadsby PJ, Lanteri-Minet M, et al. Efficacy and tolerability of erenumab in patients with episodic migraine in whom two-to-four previous preventive treatments were unsuccessful: a randomised, double-blind, placebo-controlled, phase 3B study. Lancet 2018:392:2280-7.

8 Buse DC, Manack AN, Fanning KM, et al. Chronic migraine prevalence, disability, and sociodemographic factors: results from the American migraine prevalence and prevention study. Headache 2012;52:1456-70.

9 Headache Classification Committee of the International Headache Society (IHS). The International classification of headache disorders, 3rd edition. Cephalalgia 2018;38:1-211.

10 Evers S, Afra J, Frese A, et al. EFNS guideline on the drug treatment of migraine-revised report of an EFNS task force. Eur J Neurol 2009;16:968-81.

11 Kawata AK, Hsieh R, Bender R, et al. Psychometric evaluation of a novel instrument assessing the impact of migraine on physical functioning: the migraine physical function impact diary. Headache 2017;57:1385-98.

12 Kawata AK, Hareendran A, Poon J-L. Evaluating clinically meaningful within-subject change in functioning associated with migraine prevention using the migraine physical function impact diary (MPFID). Cephalalgia 2017;37:344-5.

13 Kosinski M, Bayliss MS, Bjorner JB, et al. A six-item short-form survey for measuring headache impact: the HIT-6. Qual Life Res 2003;12:963-74.

14 Smelt AFH, Assendelft WJJ, Terwee CB, et al. What is a clinically relevant change on the HIT-6 questionnaire? an estimation in a primary-care population of migraine patients. Cephalalgia 2014;34:29-36.

15 Coeytaux RR, Kaufman JS, Chao R, et al. Four methods of estimating the minimal important difference score were compared to establish a clinically significant change in headache impact test. J Clin Epidemiol 2006:59:374-80.

16 Bayliss M, Batenhorst A. The HIT- ${ }^{\text {TM }}$ A user's guide. QualityMetric Incorporated: Lincoln, RI, 2002

17 Reilly MC, Zbrozek AS, Dukes EM. The validity and reproducibility of a work productivity and activity impairment instrument. Pharmacoeconomics 1993;4:353-65.

18 Stewart WF, Wood GC, Manack A, et al. Employment and work impact of chronic migraine and episodic migraine. J Occup Environ Med 2010;52:8-14.

19 Blumenfeld AM, Varon SF, Wilcox TK, et al. Disability, HRQoL and resource use among chronic and episodic migraineurs: results from the International burden of migraine study (IBMS). Cephalalgia 2011:31:301-15.

20 Ayer DW, Skljarevski V, Ford JH, et al. Measures of functioning in patients with episodic migraine: findings from a double-blind, randomized, placebo-controlled phase 2B trial with galcanezumab. Headache 2018;58:1225-35.

21 Skljarevski V, Matharu M, Millen BA, et al. Efficacy and safety of galcanezumab for the prevention of episodic migraine: results of the EVOLVE-2 phase 3 randomized controlled clinical trial. Cephalalgia 2018;38:1442-54.

22 Torres-Ferrus M, Alpuente A, Pozo-Rosich P. How much do calcitonin gene-related peptide monoclonal antibodies improve the quality of life in migraine? a patient's perspective. Curr Opin Neurol 2019:32:395-404. 